\title{
In vitro evaluation of antifungal effects of nanoliposomal fluconazole against fluconazole susceptible and resistant Candida species isolated from patients
}

\author{
Asadi Mehrdad ${ }^{1}$, Hashemi Seyed Jamal2* Hamishehkar Hamed ${ }^{3}$, Kordbacheh Parivash ${ }^{4}$, \\ Ghasemi Zeinab ${ }^{5}$, Mojtaba Didehdar ${ }^{6}$, Nazari Maryam ${ }^{7}$ and Sadeghi Sanam ${ }^{8}$
}

${ }^{1}$ Department of Medical Parasitology and Mycology, Faculty of Public Health, Tehran University of Medical Sciences, Tehran-Iran

${ }^{2}$ Department of Medical Parasitology and Mycology, School of Public Health, Tehran University of Medical Sciences, Tehran, Iran

${ }^{3}$ Drug Applied Research Center, Tabriz University of Medical Sciences, Tabriz, Iran

${ }^{4}$ Department of Medical Parasitology and Mycology, School of Public Health, Tehran University of Medical Sciences, Tehran, Iran

${ }^{5}$ Department of Medical Parasitology and Mycology, School of Public Health, Tehran University of Medical Sciences, Tehran, Iran

${ }^{6}$ Department of Medical Parasitology and Mycology, Arak University of Medical Sciences, Arak, Iran

${ }^{7}$ Biotechnology Research Center, Tabriz University of Medical Sciences, Tabriz, Iran.

Department of Food Science and Technology, Faculty of Agriculture, University of Tabriz, Tabriz, Iran

${ }^{8}$ Department of Nanobiotechnology, Faculty of Biological Sciences, University of Tarbiat Modares, Tehran, Iran

\section{ABSTRACT}

The aim of this study was to produce fluconazole loaded liposomal nanoparticles, to analyze their physicochemical properties and to compare their antifungal effects with the free fluconazole drug in vitro against the fluconazole susceptible and resistant Candida species isolated from patients. Six common candida species including C.albicans, C.parapsilosis, C.tropicalis, C.glabrata, C.krusei and C.guilliermondii were tested. The Liposomal nanoparticles were prepared using thin layer hydration method and soybean lecithin, cholesterol, and fluconazole at a ratio of 10: 1: 1. The nanoparticles were analyzedin terms of size, poly dispersity index, zeta potential, morphology, entrapment efficiency of drug and the amount of drug released. To investigate the antifungal effects of liposomal

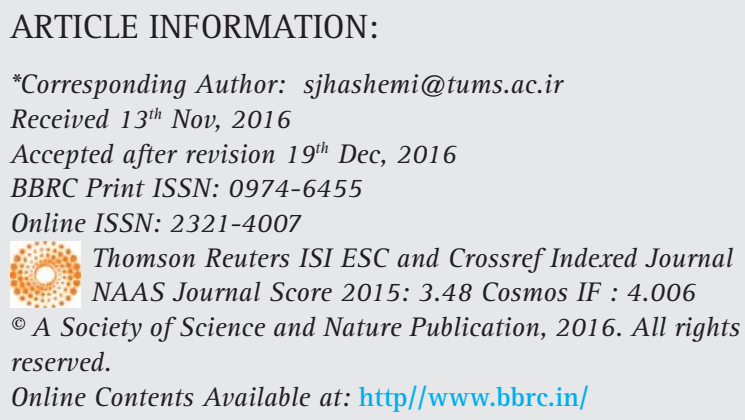


nanoparticles and compare them with the free form of fluconazole, we used Broth Microdilution as described in CLSI M27-A3.The results were analyzed using Student's T-test and indicated the greater antifungal effects of the liposomal nanoparticles containing fluconazole than the normal form of the drug. It was shown that MIC of Fluconazole was put in the range of sensitive species after exposure with the Fluconazole Nanoliposomal in most Fluconazole resistant Candida species except for the krusei species. Therefore, it is likely that we can use the new system for drug delivery to prevent drug release from the cell. In addition, this is the first research using fluconazole lipid nanoparticles against C.krusei.

KEY WORDS: NANOLIPOSOME, FLUCONAZOLE, ANTIFUNGAL ACTIVITY, CANDIDA

\section{INTRODUCTION}

Nowadays, the opportunistic pathogenic fungi are among the life threatening infections in patients with impaired immune systems. Yeasts, especially the candida species are the most common fungi that are isolated from human infections. Despite many advances in the field of health care and methods of treatment, the incidence of invasive systemic candidiasis is significantly increasing. Although Candida albicans was and is the most common agent responsible for infections in different clinical forms of candidiasis, the other species belong to the Candida types such as Candida tropicalis, Candida glabrata, Candida krusei, Candida parapsilosis, Candida guilliermondii and so on, are more or less isolated from patients. The importance of the non-albicans species has increased due to the relative resistance in some of these species like Candida tropicalis and Candida glabrata to the antifungal drugs in the recent years, (Price etal 1994, Pfaller, 1995, Wingard, 1995 Neppelenbroek et al 2006).

The increase of the different and various reports from all over the world about the drug resistance increase among the fungi and Candida species, and on the other hand, the new antifungal drugs production indicates the need for testing the susceptibility to these drugs and makes the researchers eager to determine susceptibility pattern for the various antifungal drugs, (White et al., 2002 Mohammadi et al 2016).

The side effects of anti-fungal drugs is one of the main reasons of extensive research on new anti-fungal compounds and their therapeutic effects, (FalkiewiczDulik and Macura, 2008). Meanwhile nano particles are of the particular importance, among the metal nano particles, silver nano particles has attracted many researchers due to their interesting physicochemical traits (S. Schultz et al., 2000). Recently nano particles synthesis by microbes has been considered as a suitable alternative for the mass production of nano particles, (Verma et al 2009 and Omidi et al., 2014). Solid lipid nanoparticles (SLNs) were introduced for the first time in 1991 as an alternative to common colloidal carriers such as emulsions and polymeric nanoparticles. SLNs are colloidal carriers with sizes ranging from 50 to $1000 \mathrm{~nm}$, which are formed from biolipids (Thassu et al., 2007, Yadav et al., 2013).
Recently, these particles are used as antifungal drug carriers such as Itraconazole, (Mohanty et al., 2015), ketoconazole (Souto and Muller, 2005), Griseofulvin (Aggarwal and Goindi, 2013) and Miconazole (Mendes et al., 2013). The small size of these lipid nanoparticles increases their access to the tissues and the more influence of drugs( Jenning et al., 2000). These drug delivery systems bring the controlled release of drugs, increasing chemical stability of the trapped drugs. In addition, these systems are among the safe and secure carriers that can be easily produced on a large scale (Muhlen et al., 1998 and Mehnert and Mader, 2001).

The treatment using azole antifungal agents, especially, fluconazole is introduced as an effective solution for treating infections caused by Candida, but the drug resistances have faced this treatment method with difficulties ( Revankar et al., 1996, Silva et al., 2012).

Thus, for treating these types of cases, that are not curable applying the conventional methods, the use of new treatment strategies seems to be essential. In this research, nanoliposome-containing fluconazole is prepared, and its antifungal effects are studied in vitro on the fluconazole susceptible and resistant strains of C.albicans, C.parapsilosis, C.glabrata, C.tropicalis and the resistant C.krusei species comparing to the conventional form of fluconazole.

\section{MATERIALS AND METHODS}

87 positive cultures of yeast organisms isolated from cutaneous, mucosal and systemic fungal lesions of patients referring Razi hospital in Tehran, Sina Hospital in Tabriz city and another private lab in Tabriz were collected. Clinical specimens included skin, nail, mucous discharges and the BAL samples.

Isolation of yeast: clinical samples were cultured on Sabouraud dextrose agar medium with chloramphenicol, and after the growth of yeast colonies were transferred to sterile Eppendorf tubes containing distilled water and 20\% glycerol so were kept in the freezer with $-20^{\circ}$ C.CHROMagar Candida culture medium: Yeasts isolated from patients were cultured applying linear method on the CHROMagar Candida medium in HIMEDIA Company and incubated for 48 hours at $35^{\circ} \mathrm{C}$ and then the plates 
were examined macroscopically. For the green samples testing of Candida albicans/dubliniensis, chlamydocanidia and mycelium forms production using corn meal agar medium containing Tween 80 for 48 hours at $30^{\circ}$ C was carried out.To differentiate albicans species from dubliniensis the test of chlamydoconidia production by dubliniensis species in Niger seed agar medium and the inability of C.albicans was used in the production of chlamydoconidia in the mentioned medium.

The identification of Non-Albicans candidaisolates: all of the yeasts that their colony color was not exclusive to identify them in CHROMagar medium and isolates that their color was blue and purple in CHROMagar Candida agar medium were identified using PCR-RFLP. This method is based on amplification of ribosomal DNA fragments in ITS1-5.8S-ITS2 PCR method using universal primers ITS1, ITS4 and then digestion the PCR products amplified by restriction enzyme HpaII. (figure 1, 2)

\section{PREPARATION OF LIPOSOME}

Fluconazole and cholesterol were purchased from Sigma company, and soybean lecithin was purchased from Lipoid Company. Liposomal formulation fluconazole was prepared by the method of thin layer film hydration $(\mathrm{H}$. Ola et al., 2010).

For the preparation of liposomes, lecithin, cholesterol and fluconazole were used with a ratio of 10: 1: 1. A thin layer was formed by solving these two substances in the organic solvent of chloroform-methanol (1: 1) containing fluconazole at a rate of $5.12 \mathrm{mg} / \mathrm{ml}$, then evaporation of the solvent in the rotary evaporator under the temperature of $45^{\circ} \mathrm{C}$. Then, it was hydrated

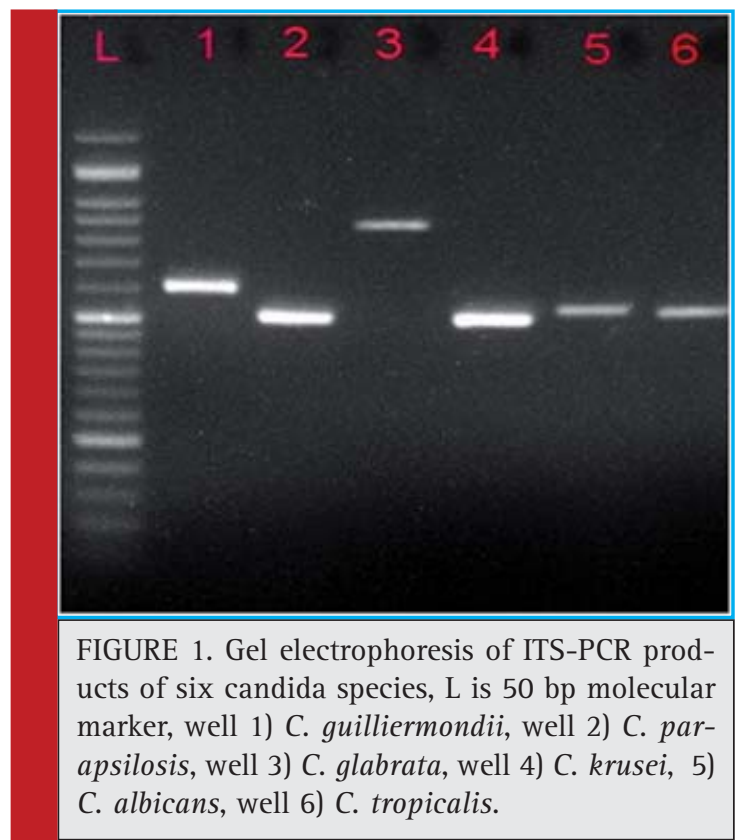

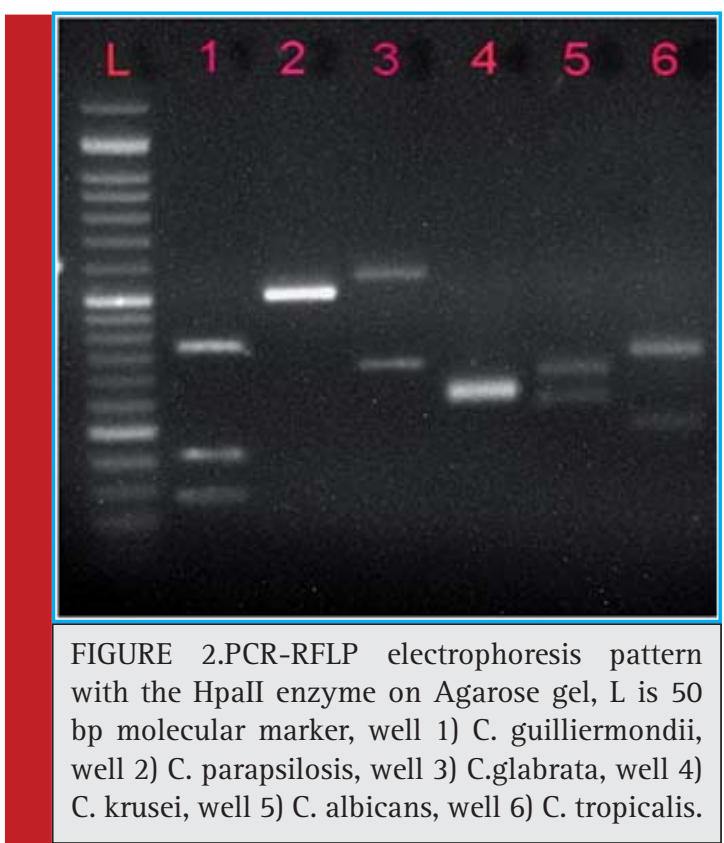

by $9 \%$ sucrose slowly at $65^{\circ} \mathrm{C}$. The homogenization action of samples was performed by a homogenizer at $20000 \mathrm{rpm}$ and ata temperature above the liposomes phase transition (70) for 10 minutes. The liposomal sample sonication operation was carried out in an ice bath. The amount of the drug that was not loaded, by the amicon filter was removed by centrifugation at $4000 \times \mathrm{g}$ for 4 minute and was passed for sterilization twice using 0/22 micron filter needle. This new formulation transferred to the laboratory and used freshly to evaluate the antifungal activity.

\section{BLANK LIPOSOMAL PREPARATION}

To prepare this formulation, it was treatedin accordance with the formulation of fluconazole liposomal formulations and the difference was that it was prepared without fluconazole.Determining the size of nanoliposomes containing fluconazole:Particle size, average diameter, nanoliposome zeta potential distribution was measured by zetasizer device based on laser light scattering. Particle size distribution in the device, was evaluated based on PDI (Poly Dispersity Index).

\section{NANOPARTICLE STRUCTURE}

For this purpose, the scanning electron microscope device was used. That a quantity of sample is placed on a glass surface with dimensions of $1 \times 1 \mathrm{~cm}$. Then it was placed inside an incubator at $37^{\circ} \mathrm{C}$ until the sample is completely dry. After that the particles were coated with gold and the images were taken with the magnification of 20,000 and 40,000. 


\section{DRUG ENTRAPMENT EFFICIENCY (DE)}

To check the encapsulated drug in the prepared liposomal formulations, the maximum absorption of fluconazole in the UV, $255 \mathrm{~nm}$ was obtained. Then, the calibration curve was drawn, and a specific amount of formulation is placed above the amicon filter and centrifuged for 5 minutes at $4{ }^{\circ} \mathrm{C}$ and $4000 \times \mathrm{g}$. A certain amount of free drug passes on the underside of Falcon removed by pipette and unloaded fluconazole absorption was measured by spectrophotometer at a wavelength of $255 \mathrm{~nm}$, and the percent of fluconazole loading was obtained by the following formula:

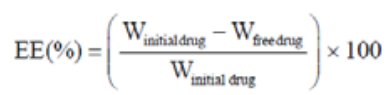

\section{ANTIFUNGAL SUSCEPTIBILITY TESTING}

In this study, the standard method of Broth Microdilution described in CLSI M27-A3-S4 is presented and the standard quality control of C. parapsilosis ATCC 22019 was used to evaluate the MIC of fluconazole and fluconazole nanolipososmal formulation (J.H. Rex et al., 2008).

To perform this test, 96-well microplateswere used. In order to dilute the fluconazole stock solution, we used the growth medium RPMI1640 (with glutamine, without bicarbonate and buffered to $\mathrm{pH}$ 7.0). To prepare this medium, we solved 10.43 grams of RPMI powder and 34.53 grams of MOPS buffer (N-MorpholinoPropanesulfoni Acid) in one liter of distilled water with gentle shaking on the flame. We used normal saline for dilution of the Nanoliposomes containing fluconazole. In the case of the two drugs, after adding to each drug the yeast cell suspension which had been prepared according to CLSI M27-A3 protocol and contained 2.5 $\times 10^{3}$ to $0.5 \times 10^{3} \mathrm{cfu} / \mathrm{ml}$ of yeast, we obtained the highest and lowest final concentrations as $64 \mu \mathrm{g} / \mathrm{ml}$ and $0.063 \mu \mathrm{lg}$ $/ \mathrm{ml}$ respectively. The well No. 12 was used as positive control. Each of the isolates was examined as dual series.

After 48 hours of incubation, to determine the Minimum Inhibitory Concentration (MIC) of fluconazole and nanoliposomal fluconazole that the fungus growing inside were not observed in the microplates and 80\% of its growth was blocked, examined and the results were read.To ensure of the non-antifungal activity of nanoliposomes particles, blank nanoparticles liposome were used in this test and no antifungal effect was observed. We have used SPSS version 18 and Student's T-test in order to analyze and compare the antifungal effects of the liposomal nanoparticles containing fluconazole and the free fluconazole.
RESULTS AND DISCUSSION

Eighty-seven positive cultures of yeast isolated from cutaneous, mucosal and systemic fungal lesions of patients referring Razi hospital in Tehran, Sina Hospital in Tabriz city and another private lab in Tabriz were collected. Due to the fact that more than one type of yeast wereisolated from some patient samples, the total number of 91 yeast colonies were isolated and tested in the next steps. The relevant results and methods of identifying can be seen in Table 1 .

The particle size of liposomal nanoparticles: In the present study, our goal was to obtain an ideal formulation in terms of particle size and the fluconazole encapsulation amount for the evaluation of the antifungal effects and its comparison with the normal form of the drug, which is reached. As can be seen, the particle size of liposome containing fluconazole is $88.9 \pm 12.1$ nm(Figure 3). Zeta potential for the fluconazole liposome formulation is $-12.20 \pm 1.88 \mathrm{mv}$ (Figure 4).

The SEM results for the nanoliposomes containing fluconazole, to study the structure and form of the nanoliposomes the scanning electron microscope was used. The results are presented in Figure 5,6. Figure 5 is captured with a lower magnification and to show a uniform distribution of particle size and Figure 6 is captured with a higher magnification to better show the structure and form of the nanoliposomes, which are spheral.

The amount of fluconazole loaded in the liposomal formulation is obtained as $75.1 \%$ in accordance with the aforementioned method.

\section{ANTIFUNGAL SUSCEPTIBILITY TESTING}

Table 2 represents the susceptibility of the examined Candida species to the fluconazole and nanoliposomal fluconazole medicines.In the present study, the produced liposomal formulation for more loading of the fluconazole drug as well as its antifungal effects on the fluconazole susceptible and resistant Candida species isolated from patients in the current form of fluconazole were created.Poly dispersity index or PDI of the prepared nanoparticles:

The results of the measurements using malvernzetasizer indicated that the formulation had a narrow particle distribution domain and the samples prepared to apply this method had a proper PDI and particle distribution. About the examination of the results of poly dispersity index or PDI of the prepared nanoparticles,it can be said that the lower the PDI, the lower the distribution of particle sizes is and the system will be more homogeneous. Generally, if PDI is in the range of 0 to 0.5 , the suspension is mono disperse, and if it is higher than 0.5 , it is poly disperse. 


\begin{tabular}{|c|c|c|c|c|c|c|}
\hline Identifying method & Percent & Quantity & Species & Total $(\%)$ & $\begin{array}{l}\text { Candida Species } \\
\text { (quantity) }\end{array}$ & $\begin{array}{l}\text { Clinical } \\
\text { species }\end{array}$ \\
\hline $\begin{array}{l}\text { Chrome Candida Agar, } \\
\text { the production of } \\
\text { chlamydocanidia }\end{array}$ & 61.4 & 56 & Candida albicans & $29(31.8)$ & $\begin{array}{l}\text { Albicans (16), } \\
\text { parapsilosis (5), } \\
\text { tropicalis (3), glabrata } \\
\text { (1), krusei (2), } \\
\text { guilliermondii (2) }\end{array}$ & Fingernail \\
\hline PCR-RFLP & 16.5 & 15 & Candida parapsilosis & $11(12)$ & $\begin{array}{l}\text { Albicans (6), parapsilosis } \\
\text { (3), tropicalis(2) }\end{array}$ & Toenail \\
\hline $\begin{array}{l}\text { Chrome Candida Agar, } \\
\text { PCR-RFLP }\end{array}$ & 8.8 & 8 & Candida tropicalis & $15(16.5)$ & $\begin{array}{l}\text { Albicans (9), parapsilosis } \\
\text { (3), tropicalis (2), krusei } \\
\text { (1) }\end{array}$ & Hand \\
\hline PCR-RFLP & 6.6 & 6 & Candida glabrata & $6(6.6)$ & $\begin{array}{l}\text { Albicans (4), } \\
\text { parapsilosis(2) }\end{array}$ & Foot \\
\hline $\begin{array}{l}\text { Chrome Candida } \\
\text { Agar,PCR-RFLP }\end{array}$ & 4.4 & 4 & Candida krusei & $19(20.9)$ & $\begin{array}{l}\text { Albicans (15), glabrata } \\
\text { (3), krusei (1) }\end{array}$ & Vagina \\
\hline \multirow[t]{2}{*}{ PCR-RFLP } & 2.2 & 2 & Candida guilliermondii & $6(6.6)$ & $\begin{array}{l}\text { Albicans (4), } \\
\text { parapsilosis(2) }\end{array}$ & Groin \\
\hline & 100 & 91 & Total & $5(5.5)$ & $\begin{array}{l}\text { Albicans (2), tropicalis } \\
\text { (1), glabrata (2) }\end{array}$ & BAL \\
\hline
\end{tabular}

Zeta potential of the fluconazole liposome formulation:

Zeta potential is an important factor in determining the stability of the colloidal system and is the best indicator to determine the status of superficial electrical dispersions. Usually, cholesterol is used to improve the stability of liposomes. Adding cholesterol increases the zeta potential of the colloidal system; as the zeta potential increases in the particles, the particles will be more stable, and on the other hand, adding cholesterol will increase the rigidity of bilayer phospholipid (Mcnaught and Wilkinson, 1997, Panyam and V. Labhasetwar, 2003).

In liposomal formulations, in the lipid composition of which there is cholesterol in addition to the lecithin, zeta potential was negative. About the stability of stearic and electrostatic combined, a minimum of $\pm 20 \mathrm{ZP}$ is desirable (Tang et al., 2014). In a research by El-Nesr et al. in 2010 on multilayer liposomes containing fluconazole, formulations with negative, neutral and positive zeta

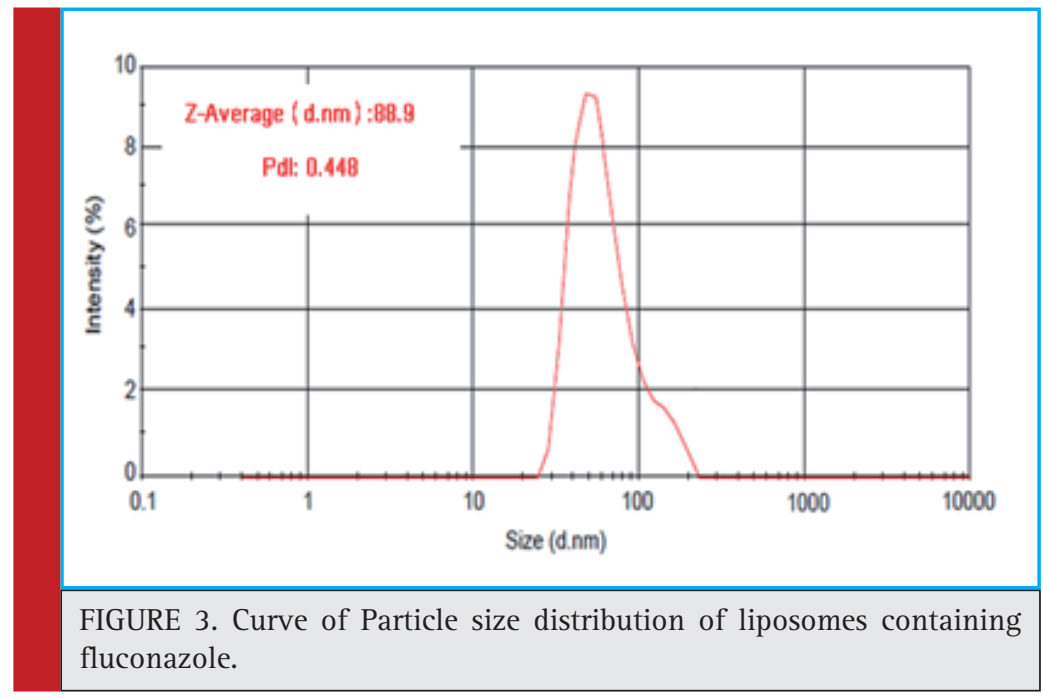




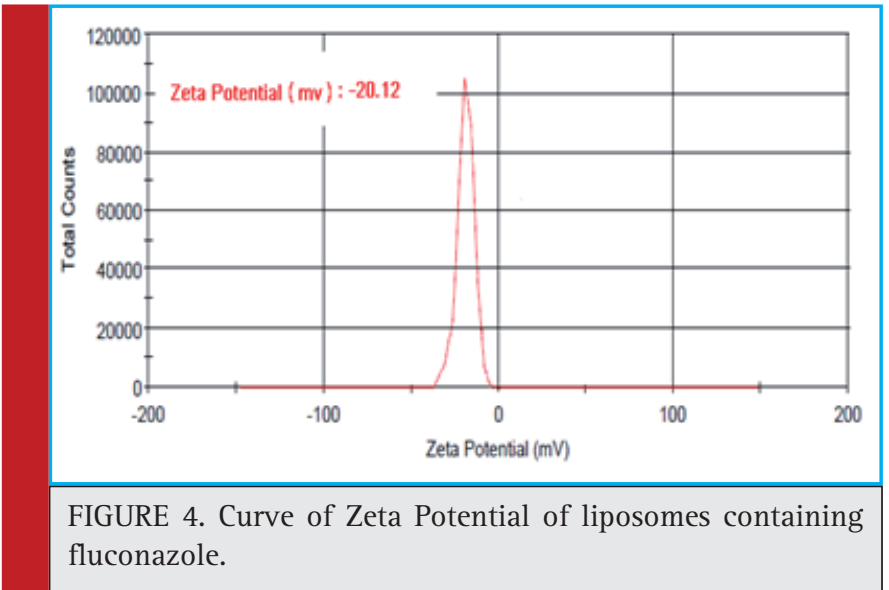

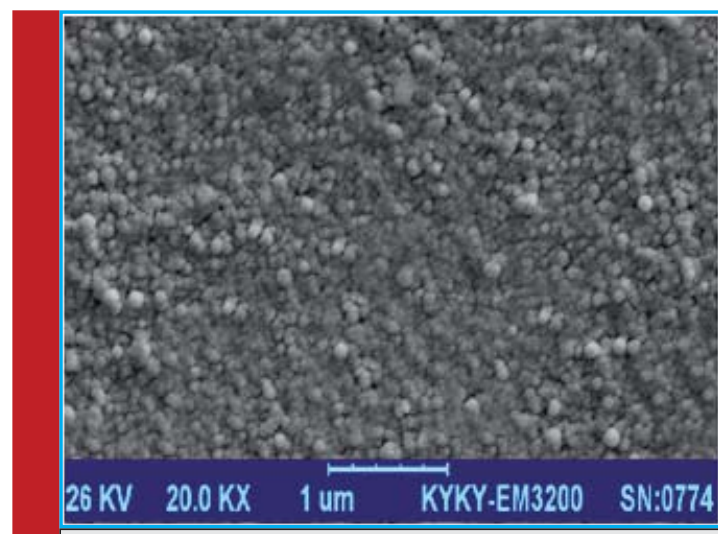

FIGURE 5. Image of nanoparticles with lower magnification.

potential were evaluated. This examination indicated that the nanoliposomes having positive, neutral and negative electric charge had the highest drug loading amount, respectively, and this can be possibly related to the electrostatic force between the positive charge of

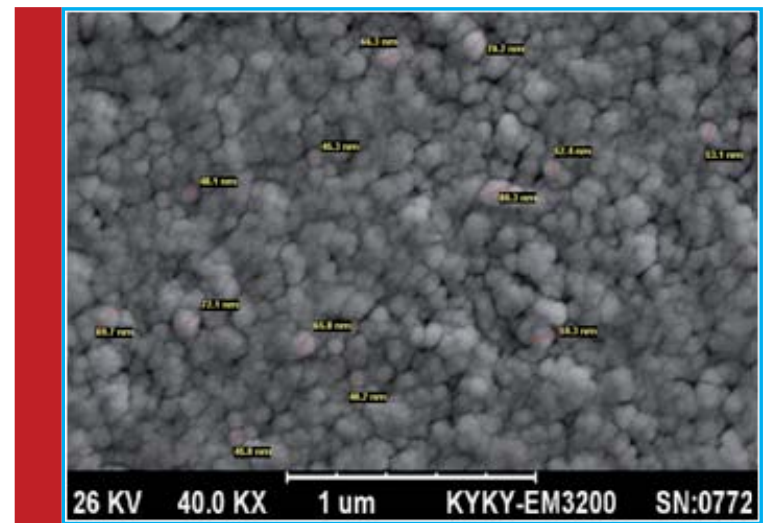

FIGURE 6. Image of nanoparticles with greater magnification. the stearylamine particles in liposome and the slightly negative charge of the fluconazole in comparison with the liposome particles with negative and neutral charge, (Ola et al., 2010).

The amount of drug loaded in the liposomal formulation of fluconazole:

The amount of drug loaded in the liposomal formulation was $75.11 \%$. In fact, a significant amount of fluconazole was encapsulated in the nanoliposomes, which is due to the hydrophilic nature of fluconazole that are not willing to be present in the external phase and as a result, a significant amount of fluconazole is encapsulated during the formation of nanoparticles.

The SEM results of nanoliposomes containing fluconazole:

In the scanning electron microscope images, the narrow range of particle distribution and the spherical shape of the formed particles was clearly observable. According to the results of SEM, the prepared particles were in the same particle size range that was obtained by nanozetasizer.

The comparison of the antifungal effects of fluconazole and liposomal nanoparticles containing fluconazole:

Table 2 shows the results of in vitro susceptibility of 6 susceptible and resistant Candida isolates to the fluconazole comparing to the fluconazole containing nanoliposome. As can be seen in the table 2, the use of the liposomal form of fluconazole significantly decreased the MIC of C.albicans species $(\mathrm{P}<0.0001)$, C.parapsilosis $(\mathrm{P}$ $<0.05)$, C.tropicalis $(\mathrm{P}=0.0005)$, C.glabrata $(\mathrm{P}=0.002)$ and C.guilliermondii $(\mathrm{P}<0.05)$, but no difference was observed in the amount of MIC of the two forms of drug in the case of C.krusei.

The $\mathrm{MIC}_{50}$ amount for C.glabrata was obtained as $8 \mu \mathrm{g} / \mathrm{ml}$, which had the highest amount of MIC relative to the nanoliposomes containing fluconazole among the isolated Candida species of patients. The resistant strain 


\begin{tabular}{|c|c|c|c|c|}
\hline \multirow{2}{*}{$\begin{array}{l}\text { Species/Antifungal } \\
\text { agent }\end{array}$} & \multirow{2}{*}{ MIC Range } & \multicolumn{3}{|c|}{$\operatorname{MIC}(\mu / \mathrm{ml})$} \\
\hline & & MIC50 & MIC90 & GM \\
\hline \multicolumn{5}{|l|}{ C. albicans (30) } \\
\hline Fluconazole & $0.5-4$ & 1 & 4 & 1.33 \\
\hline LE-Fluconazole & $0.063-4$ & 0.125 & 2 & 0.21 \\
\hline \multicolumn{5}{|l|}{ C. albicans $(26)^{*}$} \\
\hline Fluconazole & $8-64$ & 32 & 64 & 28.64 \\
\hline LE-Fluconazole & $0.063-8$ & 4 & 8 & 4.34 \\
\hline \multicolumn{5}{|l|}{ C. parapsilosis(13) } \\
\hline Fluconazole & $0.25-4$ & 1 & 2 & 0.89 \\
\hline LE-Fluconazole & $0.063-0.5$ & 0.125 & 0.45 & 0.13 \\
\hline \multicolumn{5}{|l|}{ C.parapsilosis $(2)^{*}$} \\
\hline Fluconazole & $8-32$ & 20 & -- & 16 \\
\hline LE-Fluconazole & $2-4$ & 3 & -- & 2.82 \\
\hline \multicolumn{5}{|l|}{ C.tropicalis(8) } \\
\hline Fluconazole & $0.5-2$ & 1.5 & -- & 1.29 \\
\hline LE-Fluconazole & $0.125-0.5$ & 0.25 & -- & 0.27 \\
\hline \multicolumn{5}{|l|}{ C. glabrata (5) } \\
\hline Fluconazole & $4-16$ & 8 & -- & 6.96 \\
\hline LE-Fluconazole & $1-8$ & 2 & -- & 2 \\
\hline \multicolumn{5}{|l|}{ C.glabrata $(1)^{*}$} \\
\hline Fluconazole & 64 & 64 & -- & -- \\
\hline LE-Fluconazole & 8 & 8 & -- & -- \\
\hline \multicolumn{5}{|l|}{ C.krusei $(4)^{*}$} \\
\hline Fluconazole & 64 & 64 & -- & 64 \\
\hline LE-Fluconazole & 64 & 64 & -- & 64 \\
\hline \multicolumn{5}{|l|}{ C.guilliermondii(2) } \\
\hline Fluconazole & 2 & 2 & -- & 2 \\
\hline LE-Fluconazole & 0.125 & 0.125 & -- & 0.125 \\
\hline \multicolumn{5}{|l|}{ C.parapsilosis 22019} \\
\hline Fluconazole & 0.5 & 0.5 & -- & -- \\
\hline LE-Fluconazole & 0.125 & 0.125 & -- & -- \\
\hline
\end{tabular}

to the fluconazole of C.glabrata which has MIC equal to $64 \mu \mathrm{g} / \mathrm{ml}$, had 4 times decrease in the MIC and placed in the susceptible species after exposing to the fluconazole liposomal form. The amount of $\mathrm{MIC}_{50}$ for the fluconazole susceptible strains of C.albicans, C.parapsilosis, C.tropicalis, and C.guilliermondii against to the fluconazole liposomal form, were $1 \mu \mathrm{g} / \mathrm{ml}, 1 \mu \mathrm{g} / \mathrm{ml}, 1.5 \mu \mathrm{g} / \mathrm{ml}$ and $2 \mu \mathrm{g} / \mathrm{ml}$, respectively. The amount of $\mathrm{MIC}_{50}$ for the resistant strains of C.albicans, C.parapsilosis, C.krusei were $3 \mu \mathrm{g} / \mathrm{ml}, 4 \mu \mathrm{g} / \mathrm{ml}$ and $64 \mu \mathrm{g} / \mathrm{ml}$, respectively.

There is a high possibility that the reason for the low amount of MIC in the fluconazole resistant Can- dida strains relative to the nanoliposomal fluconazole is related to the identified resistance mechanisms of pathogenic fungi to drugs; the mechanisms that make fungi survive in the vicinity of a series of toxic substrates as well as antifungal drugs( Mishra et al., 2007, Morschhäuser, 2010). Various molecular mechanisms have been identified in the development of strains resistant to antifungal drugs (Franz et al., 1998), that these mechanisms include reducing the transmission and diffusion of the drug into the cell, changes in the enzymes of the pathway of ergosterol Biosynthesis, changes in the target enzyme (point mutations, expression increase 
and gene change) and the increase of drug release to the outside by membrane diffusion pumps, activation of the enzymes that break down drugs outside the cell wall (Favre et al., 1999, Hitchcock et al., 1990, Li et al., 2004, Löffler et al., 1997, Sanglard and Odds, 2002, White et al., 1998).

From the mentioned cases, the most important reason for the resistance of Candida to the azole drugs is related to the expression of the high amount of transporter membrane proteins that lead to the pumping of azoles to the outside of the cell. The result is the reduction of drug concentration in cell and in this case, the drug concentration inside the cell is under the necessary level needed for creating the inhibitory effect on Erg11p (Caira et al., 2004). Two main types of drug release pumps from the fungus cells causes their resistance to drugs: ABS and MFS (Lamping et al., 2010). Cdr1 and Cdr2 are among the important transporters of the drug to the outside of the cell in C.albicans, C.glabrata and C.parapsilosis and the increase in their expression leads to the release of drug from the cell, which finally leads to the resistance of mentioned species to the fluconazole (White et al., 2002, Bennett et al., 2004 and Souza et al., 2015).

In the examination conducted by us, the lipid nanoparticles are fused quickly with the cell membrane of yeast due to the hydrophobic outer surface, and as they are small in terms of size, they are capable of penetrating to the yeast cell and causes the drug protection against enzymes and efflux pumps. Also, the lipid drug delivery systems due to the use biodegradable lipids are low toxic, have the ability to get lyophilized (Garud et al., 2012) and are capable of controlled release of the drugs, (Bose et al., 2013).

In a recent study conducted by Moazeni et al. in 2016, using lipid nanoparticles against fluconazole susceptible and resistant species in vitro, the results indicated the satisfying effect of lipid nanoparticles containing fluconazole on the resistant strains of C.albicans, C.parapsilosis and C.glabrata (M. Moazeni et al., 2016).

Similarly, Gupta et al. had observed that the use of drug delivery system can lead to the increase of fluconazole penetration from the skin surface (Gupta et al., 2013). As the fluconazole prevents the synthesis of ergosterol by affecting the $\alpha$-demethylase 14 enzyme, in the investigations of Venkateswarlu et al. it was determined that the $\alpha$-demethylase 14 existing in the C.krusei differs the $\alpha$-demethylase 14 of C.albicans (Venkateswarlu et al., 1997, Venkateswarlu et al., 1996).

Studies by Orozco et al. indicated that the intracellular accumulation of fluconazole of C.krusei and C.albicans are similar (Orozco et al., 1998). This in turn could explain the reason why C.krusei was resistant to the fluconazole exposure as well as the form of fluconazole nanoparticles.
In the current study, the function of a new drug delivery system was evaluated on the fluconazole resistant and susceptible Candida strains isolated from the patients. Although different mechanisms of resistance to fluconazole are expressed, it seems that the resistance is due to the pumps releasing drug from the cell and it is one of the main reasons for these resistances. Therefore, it is likely that we can use the new system for drug delivery to prevent drug release from the cell. In addition, this is the first research using fluconazole lipid nanoparticles against C.krusei.

\section{REFERENCES}

Aggarwal, N. and S. Goindi (2013) Preparation and in vivo evaluation of solid lipid nanoparticles of griseofulvin for dermal use. Journal of biomedical nanotechnology Vol. 9 No 4: Page 564-576.

Bennett, J.E., K. Izumikawa and K.A. Marr (2004) Mechanism of increased fluconazole resistance in Candida glabrata during prophylaxis. Antimicrobial Agents and Chemotherapy Vol. 48 No 5: Page 1773-7.

Bose, S., Y. Du, P. Takhistov and B. Michniak-Kohn (2013) Formulation optimization and topical delivery of quercetin from solid lipid based nanosystems. International Journal of Pharmaceutics Vol. 441 No 1-2: Page 56-66.

Caira, M.R., K.A. Alkhamis and R.M. Obaidat (2004) Preparation and crystal characterization of a polymorph, a monohydrate, and an ethyl acetate solvate of the antifungal fluconazole. Journal of Pharmaceutical Sciences Vol. 93 No 3: Page 601-11.

Falkiewicz-Dulik, M. and A. Macura (2008) Nanosilver as substance biostabilising footwear materials in the foot mycosis prophylaxis. Mikologia Lekarska Vol. 15 No: Page 145-150.

Favre, B., M. Didmon and N.S. Ryder (1999) Multiple amino acid substitutions in lanosterol $14 \alpha$-demethylase contribute to azole resistance in Candida albicans. Microbiology Vol. 145 No 10: Page 2715-2725.

Franz, R., S.L. Kelly, D.C. Lamb, D.E. Kelly, M. Ruhnke and J. Morschhauser (1998) Multiple molecular mechanisms contribute to a stepwise development of fluconazole resistance in clinical Candida albicans strains. Antimicrobial Agents and Chemotherapy Vol. 42 No 12: Page 3065-72.

Garud, A., D. Singh and N. Garud (2012) Solid Lipid Nanoparticles (SLN): Method, Characterization and Applications. International Current Pharmaceutical Journal Vol. 1 No 11: Page 384-393.

Gupta, M., S. Tiwari and S.P. Vyas (2013) Influence of various lipid core on characteristics of SLNs designed for topical delivery of fluconazole against cutaneous candidiasis. Pharmaceutical development and technology Vol. 18 No 3: Page 550-559.

Hitchcock, C.A., K. Dickinson, S. Brown, E. Evans and D. Adams (1990) Interaction of azole antifungal antibiotics with 
cytochrome P-450-dependent $14 \alpha$-sterol demethylase purified from Candida albicans. Biochemical Journal Vol. 266 No 2: Page 475-480.

Jenning, V., M. Schäfer-Korting and S. Gohla (2000) Vitamin A-loaded solid lipid nanoparticles for topical use: drug release properties. Journal of controlled release Vol. 66 No 2: Page 115-126.

Lamping, E., P.V. Baret, A.R. Holmes, B.C. Monk, A. Goffeau and R.D. Cannon (2010) Fungal PDR transporters: Phylogeny, topology, motifs and function. Fungal Genetics and Biology Vol. 47 No 2: Page 127-42.

Li, X., N. Brown, A.S. Chau, J.L. Lopez-Ribot, M.T. Ruesga, G. Quindos, C.A. Mendrick, R.S. Hare, D. Loebenberg, B. Didomenico and P.M. Mcnicholas (2004) Changes in susceptibility to posaconazole in clinical isolates of Candida albicans. The Journal of antimicrobial chemotherapy Vol. 53 No 1: Page 74-80.

Löffler, J., S.L. Kelly, H. Hebart, U. Schumacher, C. Lass-Flörl and H. Einsele (1997) Molecular analysis of cyp51 from fluconazole-resistant Candida albicans strains. FEMS microbiology letters Vol. 151 No 2: Page 263-268.

Mcnaught, A.D. and A. Wilkinson (1997)Compendium of chemical terminology (Vol. 1669). Blackwell Science,Oxford.

Mehnert, W. and K. Mader (2001) Solid lipid nanoparticles: production, characterization and applications. Advanced Drug Delivery Reviews Vol. 47 No 2-3: Page 165-96.

Mendes, A.I., A.C. Silva, J.A. Catita, F. Cerqueira, C. Gabriel and C.M. Lopes (2013) Miconazole-loaded nanostructured lipid carriers (NLC) for local delivery to the oral mucosa: improving antifungal activity. Colloids Surf B Biointerfaces Vol. 111 No: Page 755-63.

Mishra, N.N., T. Prasad, N. Sharma, A. Payasi, R. Prasad, D.K. Gupta and R. Singh (2007) Pathogenicity and drug resistance in Candida albicans and other yeast species. A review. Acta Microbiologica et Immunologica Hungarica Vol. 54 No 3: Page 201-35.

Moazeni, M., H.R. Kelidari, M. Saeedi, K. Morteza-Semnani, M. Nabili, A.A. Gohar, J. Akbari, E. Lotfali and A. Nokhodchi (2016) Time to overcome fluconazole resistant Candida isolates: Solid lipid nanoparticles as a novel antifungal drug delivery system. Colloids and Surfaces B: Biointerfaces Vol. 142 No: Page 400-407.

Mohammadi, F., P. Dehghan, S. Nekoeian and S.J. Hashemi (2016) Determination of antifungal susceptibility patterns among the environmental isolates of Aspergillus fumigatus in Iran. Advanced biomedical research Vol. 5 No: Page.

Mohanty, B., D.K. Majumdar, S.K. Mishra, A.K. Panda and S. Patnaik (2015) Development and characterization of itraconazole-loaded solid lipid nanoparticles for ocular delivery. Pharmaceutical Development and Technology Vol. 20 No 4: Page 458-64.

Morschhäuser, J. (2010) Regulation of multidrug resistance in pathogenic fungi. Fungal Genetics and Biology Vol. 47 No 2: Page 94-106.
Neppelenbroek, K., N. Campanha, D.M.P. Spolidório, L.C. Spolidorio, R. Seó and A.C. Pavarina (2006) Molecular fingerprinting methods for the discrimination between C. albicans and $C$. dubliniensis. Oral diseases Vol. 12 No 3: Page 242-253.

Ola, H., S.A. Yahiya and O.N. El-Gazayerly (2010) Effect of formulation design and freeze-drying on properties of fluconazole multilamellar liposomes. Saudi pharmaceutical journal Vol. 18 No 4: Page 217-224.

Omidi, B., S.J. Hashemi, M. Bayat and K. Larijani (2014) Biosynthesis of Silver Nanoparticles by Lactobacillus fermentum. Bulletin of Environment, Pharmacology and Life Sciences Vol. 3 No: Page 186-192.

Orozco, A.S., L.M. Higginbotham, C.A. Hitchcock, T. Parkinson, D. Falconer, A.S. Ibrahim, M.A. Ghannoum and S.G. Filler (1998) Mechanism of Fluconazole Resistance in Candida_krusei. Antimicrobial agents and chemotherapy Vol. 42 No 10: Page 2645-2649.

Panyam, J. and V. Labhasetwar (2003) Biodegradable nanoparticles for drug and gene delivery to cells and tissue. Advanced Drug Delivery Reviews Vol. 55 No 3: Page 329-47.

Pfaller, M.A. (1995) Epidemiology of candidiasis. J Hosp Infect Vol. 30 Suppl No: Page 329-38.

Price, M.F., M.T. Larocco and L.O. Gentry (1994) Fluconazole susceptibilities of Candida species and distribution of species recovered from blood cultures over a 5-year period. Antimicrobial Agents and Chemotherapy Vol. 38 No 6: Page 1422-4.

Revankar, S.G., W.R. Kirkpatrick, R.K. Mcatee, O.P. Dib, A.W. Fothergill, S.W. Redding, M.G. Rinaldi and T.F. Patterson (1996) Detection and significance of fluconazole resistance in oropharyngeal candidiasis in human immunodeficiency virusinfected patients. Journal of Infectious Diseases Vol. 174 No 4: Page 821-7.

Rex, J.H., B. Alexander, D. Andes, B. Arthington-Skaggs, S. Brown, V. Chaturveli, A. Espinel-Ingroff, M. Ghannoum, C. Knapp and M. Motyl (2008) Reference method for broth dilution antifungal susceptibility testing of filamentous fungi: approved standard. Vol. No: Page.

Sanglard, D. and F.C. Odds (2002) Resistance of Candida species to antifungal agents: molecular mechanisms and clinical consequences. Lancet Infectious Diseases Vol. 2 No 2: Page 73-85.

Schultz, S., D.R. Smith, J.J. Mock and D.A. Schultz (2000) Single-target molecule detection with nonbleaching multicolor optical immunolabels. Proceedings of the National Academy of Sciences of the United States of America Vol. 97 No 3: Page 996-1001.

Silva, S., M. Negri, M. Henriques, R. Oliveira, D.W. Williams and J. Azeredo (2012) Candida glabrata, Candida parapsilosis and Candida tropicalis: biology, epidemiology, pathogenicity and antifungal resistance. FEMS Microbiology Reviews Vol. 36 No 2: Page 288-305.

Souto, E.B. and R.H. Muller (2005) SLN and NLC for topical delivery of ketoconazole. Journal of Microencapsulation Vol. 22 No 5: Page 501-10. 
Souza, A.C., B.B. Fuchs, H.M. Pinhati, R.A. Siqueira, F. Hagen, J.F. Meis, E. Mylonakis and A.L. Colombo (2015) Candida parapsilosis Resistance to Fluconazole: Molecular Mechanisms and In Vivo Impact in Infected Galleria mellonella Larvae. Antimicrobial Agents and Chemotherapy Vol. 59 No 10: Page 6581-7.

Tang, X., H. Zhu, L. Sun, W. Hou, S. Cai, R. Zhang and F. Liu (2014) Enhanced antifungal effects of amphotericin B-TPGSb-(PCL-ran-PGA) nanoparticles in vitro and in vivo. International journal of nanomedicine Vol. 9 No: Page 5403.

Thassu, D., M. Deleers and Y.V. Pathak (2007)Nanoparticulate drug delivery systems. CRC Press.

Venkateswarlu, K., D.W. Denning and S.L. Kelly (1997) Inhibition and interaction of cytochrome P450 of Candida krusei with azole antifungal drugs. Journal of Medical and Veterinary Mycology Vol. 35 No 1: Page 19-25.

Venkateswarlu, K., D.W. Denning, N.J. Manning and S.L. Kelly (1996) Reduced accumulation of drug in Candida krusei accounts for itraconazole resistance. Antimicrobial Agents and Chemotherapy Vol. 40 No 11: Page 2443-6.

Verma, V., R. Kharwar and A. Gange (2009) Biosynthesis of noble metal nanoparticles and their application. CAB Reviews:
Perspectives in Agriculture, Veterinary Science, Nutrition and Natural Resources Vol. 4 No 026: Page 1-17.

White, T.C., S. Holleman, F. Dy, L.F. Mirels and D.A. Stevens (2002) Resistance mechanisms in clinical isolates of Candida albicans. Antimicrobial Agents and Chemotherapy Vol. 46 No 6: Page 1704-13.

White, T.C., K.A. Marr and R.A. Bowden (1998) Clinical, cellular, and molecular factors that contribute to antifungal drug resistance. Clinical microbiology reviews Vol. 11 No 2: Page 382-402.

Wingard, J.R. (1995) Importance of Candida species other than C. albicans as pathogens in oncology patients. Clinical Infectious Diseases Vol. 20 No 1: Page 115-125.

Yadav, N., S. Khatak and U.V.S. Sara (2013) Solid lipid nanoparticles-a review. International Journal of Applied Pharmaceutics Vol. 5 No 2: Page 8-18.

Zur Muhlen, A., C. Schwarz and W. Mehnert (1998) Solid lipid nanoparticles (SLN) for controlled drug delivery-drug release and release mechanism. European Journal of Pharmaceutics and Biopharmaceutics Vol. 45 No 2: Page 149-55. 